\title{
Erratum: Eli, B., et al. Depression in Children and Adolescents on the Qinghai-Tibet Plateau: Associations with Resilience and Prosocial Behavior. Int. J. Environ. Res. Public Health 2021, 18, 440
}

\author{
Buzohre Eli ${ }^{1,2} \mathbb{D}^{\mathbb{D}}$, Yueyue Zhou ${ }^{1,2} \mathbb{D}$, Yiming Liang ${ }^{1,2} \mathbb{D}$, Jin Cheng ${ }^{3}$, Jiazhou Wang ${ }^{1,2} \mathbb{D}$, Changbing Huang ${ }^{4}$, \\ Xi Xuan ${ }^{5}$ and Zhengkui Liu ${ }^{1,2, *}$
}

1 CAS Key Laboratory of Mental Health, Institute of Psychology, Chinese Academy of Sciences, Beijing 100101, China; ail@psych.ac.cn (B.E.); zhouyueyue@psych.ac.cn (Y.Z.); liangym@psych.ac.cn (Y.L.); wangjz@psych.ac.cn (J.W.)

2 Department of Psychology, University of Chinese Academy of Sciences, Beijing 100049, China

3 School of Psychology, Beijing Sport University, Beijing 100084, China; chengjin2020@163.com

4 CAS Key Laboratory of Behavioral Science, Institute of Psychology, Chinese Academy of Sciences, Beijing 100101, China; huangcb@psych.ac.cn

5 Department of Law and Politics, Nankai University Binhai College, Tianjin 300270, China; xuanxi123456@126.com

* Correspondence: liuzk@psych.ac.cn; Tel.: +86-10-64871358; Fax: +86-10-64872070

check for updates

Citation: Eli, B.; Zhou, Y.; Liang, Y.; Cheng, J.; Wang, J.; Huang, C.; Xuan, X.; Liu, Z. Erratum: Eli, B., et al. Depression in Children and Adolescents on the Qinghai-Tibet Plateau: Associations with Resilience and Prosocial Behavior. Int. J. Environ. Res. Public Health 2021, 18, 440. Int. J. Environ. Res. Public Health 2021, 18, 3707. https://doi.org/10.3390/ ijerph18073707

Received: 26 March 2021 Accepted: 1 April 2021

Published: 2 April 2021

Publisher's Note: MDPI stays neutral with regard to jurisdictional claims in published maps and institutional affiliations.

Copyright: (c) 2021 by the authors. Licensee MDPI, Basel, Switzerland. This article is an open access article distributed under the terms and conditions of the Creative Commons Attribution (CC BY) license (https:// creativecommons.org/licenses/by/ $4.0 /)$.

\section{Incorrect Text}

There was a small mistake in the sixth paragraph of the Discussion: the positions of two words ("positively" and "negatively") should be exchanged. The correct expression [1] is "In addition, we also found that the education levels of the parents were positively associated with depression but negatively associated with both resilience and prosocial behavior."

We apologize for any inconvenience caused to the readers by this mistake.

Funding: This work was supported by the Tianjin Program in philosophy and social sciences (TJJX19008) and the poverty alleviation program of the Chinese Academy of Sciences (KFJ-FP-201,906).

Institutional Review Board Statement: The survey protocol was approved by the ethics review committee of the Institute of Psychology, Chinese Academy of Sciences (project identification code: H16014).

Informed Consent Statement: Informed consent was obtained from all participants involved in the study.

Conflicts of Interest: The authors declare that they have no conflicts of interest.

\section{Reference}

1. Eli, B.; Zhou, Y.; Liang, Y.; Cheng, J.; Wang, J.; Huang, C.; Xuan, X.; Liu, Z. Depression in Children and Adolescents on the Qinghai-Tibet Plateau: Associations with Resilience and Prosocial Behavior. Int. J. Environ. Res. Public Health 2021, 18, 440. [CrossRef] [PubMed] 